ARTICLE

\title{
Ultra-responsive soft matter from strain-stiffening hydrogels
}

Maarten Jaspers', Matthew Dennison², Mathijs F.J. Mabesoone', Frederick C. MacKintosh², Alan E. Rowan ${ }^{1} \&$ Paul H.J. Kouwer ${ }^{1}$

The stiffness of hydrogels is crucial for their application. Nature's hydrogels become stiffer as they are strained. This stiffness is not constant but increases when the gel is strained. This stiffening is used, for instance, by cells that actively strain their environment to modulate their function. When optimized, such strain-stiffening materials become extremely sensitive and very responsive to stress. Strain stiffening, however, is unexplored in synthetic gels since the structural design parameters are unknown. Here we uncover how readily tuneable parameters such as concentration, temperature and polymer length impact the stiffening behaviour. Our work also reveals the marginal point, a well-described but never observed, critical point in the gelation process. Around this point, we observe a transition from a low-viscous liquid to an elastic gel upon applying minute stresses. Our experimental work in combination with network theory yields universal design principles for future strain-stiffening materials.

\footnotetext{
${ }^{1}$ Department of Molecular Materials, Radboud University Nijmegen, Institute for Molecules and Materials, Heyendaalseweg 135, 6525 Nijmegen, The Netherlands. ${ }^{2}$ Department of Physics and Astronomy VU University, De Boelelaan, 1081 Amsterdam, The Netherlands. Correspondence and requests for materials should be addressed to A.E.R. (email: a.rowan@science.ru.nl) or to P.H.J.K. (email: p.kouwer@science.ru.nl).
} 
M echano-responsive gels are very common in nature; for instance, gels based on actin, collagen, fibrin, intermediate filaments and many more proteins and carbohydrates display mechanical properties that are not only dependent on their biochemical environment, but also show an immediate response to deformation ${ }^{1,2}$. Many of these gels strainstiffen (they become stiffer as the stress or strain in the material increases), which, for instance, aids in the protection of tissues from rupture and in long-distance cell-cell communication ${ }^{1,3}$. Typically, these biopolymers show a universal structural design element: they are relatively stiff and assemble into bundles or fibrils of defined dimensions, which results in both a high sensitivity and a high responsiveness towards stress.

In contrast to these biogels, synthetic hydrogels, including many artificial extracellular matrices are generally not (or at most very weakly) responsive to stress, that is, their stiffness is constant over the entire relevant stress range $e^{4,5}$. If stiffening behaviour is observed at all, it is found at high stresses; for (soft) hydrogels, this is commonly far beyond the rupture stress of the material. It is surprising that, despite the general consensus on the crucial role of mechanics in biomedical processes ${ }^{5-10}$, the stiffening aspect, and in particular the difference in behaviour of biological and synthetic gels has remained largely overlooked ${ }^{11-13}$. As a result, the relation between common network parameters and conditions with mechanical responsiveness are only available from studies on biopolymer gels ${ }^{1,2,14}$ (with highly limited parameter space) or from theory and simulations ${ }^{15}$.

Recently, we reported a fully synthetic gel that uniquely stiffens in the same stress and strain regime as biogels ${ }^{16}$. In this paper we exploit the broad scope for manipulation that synthetic materials offer, and we describe the effect of common, easily controllable variables (concentration, polymer length and temperature) on the mechanical properties of semi-flexible polymer hydrogels. We show for instance that at sufficient stress, a universal mechanical response is observed, which means that sometimes, counterintuitively, stiffer materials can be obtained at lower polymer concentrations. Moreover, the thermoresponsive nature of our gel allows us to precisely control the crosslink density, a tool to enter the marginal regime that is the regime where the fluid polymer solution turns into an elastic gel. Our work in this experimentally unexplored regime agrees quantitatively with theory and simulation results of semi-flexible networks ${ }^{17}$. This excellent agreement emphasizes that our results are not specific for our polymer gels only, but will be generally applicable for the design of other mechano-responsive soft matter.

\section{Results}

Materials. The biomimetic strain-stiffening hydrogels are based on ethyleneglycol-functionalized polyisocyanopeptides $\mathbf{P 1 a}-\mathbf{g}$ (PICs, Fig. 1). The polymers were synthesized and characterized using a slightly modified literature procedure (see Methods section $)^{16,18}$. Variation of the monomer/catalyst ratio in the



Figure 1 | Ethylene glycol substituted polyisocyanopeptides. Molecular structure of the tri(ethylene glycol)-substituted polyisocyanopeptides. In purple the polymer backbone of 270-1,430 repeat units. Every carbon atom holds a substituent, composed of a dipeptide (Ala-Ala, red) and a short methyl-terminated tri(ethylene glycol) tail (blue). reaction mixture gave control over the polymer length (Table 1), which was characterized by viscometry and atomic force microscopy (AFM) studies (Supplementary Fig. 1) since standard polymer length characterization techniques based on separation by gel permeation chromatography or field-flow fractionation gave unreliable results (poor separation), possibly due to the high backbone stiffness. We used viscometry data (available over the entire molecular weight range) in combination with the Mark - Houwink equation (Methods section) to determine molecular weights and with AFM data to determine distributions. As such, we prepared seven polymers with molecular weights ranging from $M_{\mathrm{v}}=80-450 \mathrm{~kg} \mathrm{~mol}^{-1}$, equivalent to average contour lengths $L$ of $30-180 \mathrm{~nm}$ (ref. 19). To prepare a hydrogel, a small amount of polymer (typically $1 \mathrm{mg}$ ) is dissolved in cold water $(1 \mathrm{ml})$ by stirring for $24 \mathrm{~h}$ in a cold room at $4{ }^{\circ} \mathrm{C}$ and subsequently heating the solution beyond the gelation temperature, which is polymer length and (weakly) concentration dependent. The thermoreversible gelation process is the result of hydrophobic effects of the oligo(ethyleneglycol) tails ${ }^{20}$. At the lower critical solution temperature, the polymer becomes hydrophobic and forms the entangled bundles of polymer chains of which the hydrogel is composed.

Mechanical analysis. In strain-stiffening materials, the plateau shear modulus $G_{0}$, defined as the ratio between the stress $\sigma$ and the strain $\gamma$, is not a constant but increases with increasing stress (or strain) after a critical stress $\sigma_{c}$ is reached (Fig. 2a). To more accurately describe the mechanical behaviour, we use the differential modulus $K^{\prime}=\partial \sigma / \partial \gamma$ and define a low stress linear regime where $K^{\prime}=G_{0}$ and a higher stress stiffening regime where the modulus depends on $\sigma$ (Fig. 2b). This introduces two additional important parameters: the critical stress $\sigma_{\mathrm{c}}$ and stiffening index $m$ (Fig. 2b). The former is the stress onset for nonlinearity and determines the sensitivity of the material; a low $\sigma_{\mathrm{c}}$ yields a high mechanical sensitivity. The latter index $m$ represents the (intensity of the) stress response. Indeed, biological gels are commonly characterized by these three parameters. For instance, a typical actin gel has a high plateau modulus, a low critical stress (very sensitive to small stresses) and a high stiffening index (large response $)^{2}$. Contrarily, a fibrin gel at similar concentration has a much lower $G_{0}$, a higher $\sigma_{c}$ and a stiffening index that is a function of the applied stress resulting from the hierarchical organization of the protein assembly ${ }^{21}$.

The experimental data of the P1f gel (Fig. 2a,b) shows that for the PIC hydrogels, $\sigma_{\mathrm{c}}$ lies well into the biologically accessible

\begin{tabular}{|c|c|c|c|c|}
\hline & $M_{v}\left(\mathrm{~kg} \mathrm{~mol}^{-1}\right)$ & PDI $^{\star}$ & $\boldsymbol{n}^{\dagger}$ & $L^{\ddagger}(\mathbf{n m})$ \\
\hline P1a & 86 & - & 272 & 34 \\
\hline P1b & 140 & - & 443 & 55 \\
\hline P1c & 195 & 1.3 & 617 & 77 \\
\hline P1d & 277 & 1.4 & 877 & 110 \\
\hline P1e & 339 & 1.4 & 1073 & 134 \\
\hline P1f & 400 & 1.4 & 1266 & 158 \\
\hline P1g & 452 & 1.3 & 1430 & 179 \\
\hline \multicolumn{5}{|c|}{$\begin{array}{l}\text { AFM, atomic force microscopy; PDI, polymer dispersity index } \\
\star \text { The PDI, showing that the molecular weight distribution of the polymers was similar for all } \\
\text { materials and was determined by statistical analysis of the AFM images. The PDIs of the shorter } \\
\text { polymers could not reliably be obtained using this method. } \\
\text { †Degree of polymerization. } \\
\begin{array}{l}\ddagger \\
\text { the calculated polymer contour length is based on the dimensions of the helical structure of } \\
\text { the polymer }\end{array} \text {. As an example, the viscosity molecular weight } M_{v}=400 \mathrm{~kg} \mathrm{~mol}^{-1} \text { of P1f } \\
\text { corresponds to chains of } n \approx 1,300 \text { monomers with a contour length } L \approx 160 \mathrm{~nm} \text {. }\end{array}$} \\
\hline
\end{tabular}


a

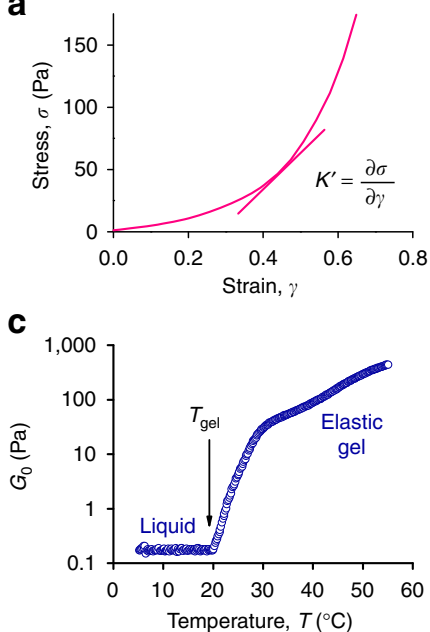

b

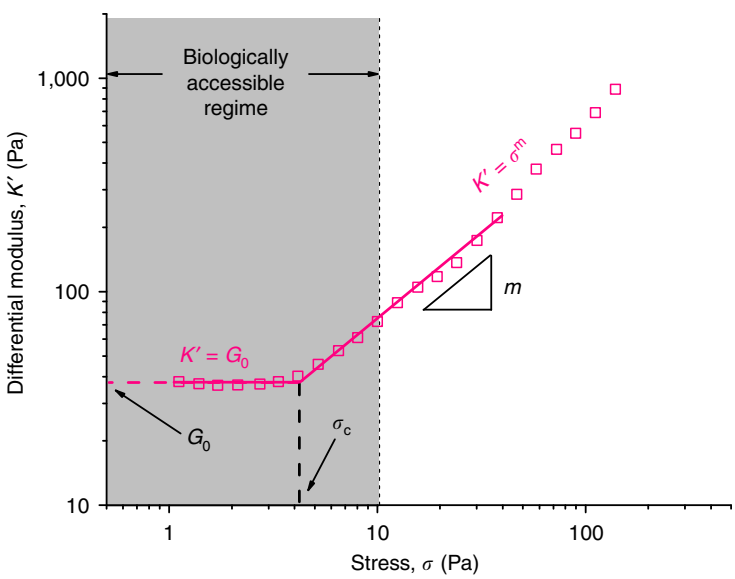

Figure 2 | Strain stiffening of a polyisocyanopeptide hydrogel. (a) Stress-strain curve of polymer P1f $\left(1 \mathrm{mg} \mathrm{ml} \mathbf{m}^{-1}, T^{\prime}=37^{\circ} \mathrm{C}\right)$ in a stress ramp. (b) The stiffness represented as the differential modulus $K^{\prime} \equiv \partial \sigma / \partial \gamma$ as a function of stress $\sigma$ for the same polymer. At low stress, $K^{\prime}=G_{0}$ the plateau modulus, but beyond a critical stress $\sigma_{c^{\prime}} K^{\prime}$ increases, following $K^{\prime} \propto \sigma^{m}$ where the exponent $m$ is the stiffening index. (c) The thermally induced gelation process is unmistakably observed by plotting $G_{0}$ versus temperature $T$. We define the gel point as the onset of modulus increase, here at $19^{\circ} \mathrm{C}$.

range $^{13}$ (the forces that cells can apply to their matrix) and that the response to stress and other external factors such as temperature (Fig. 2c) is large. In the following paragraphs, we demonstrate how the mechanical parameters stiffness $G_{0}$, sensitivity $\sigma_{\mathrm{c}}$ and responsiveness $m$ can be controlled by the variables temperature $T$, polymer length $L$ and concentration $c$. Can one, for instance, independently tune $G_{0}$ and $\sigma_{\mathrm{c}}$ to give stiffer, but nonetheless more sensitive materials? How do $T, L$ and $c$ impact the strength of the response? Or more generally, what are the important design parameters to create highly mechanically responsive materials and how should one access these highly responsive regimes?

We performed macroscopic rheology to determine the mechanical properties of the hydrogels in the linear and the nonlinear regime. The nonlinear regime was studied using a prestress protocol, where the sample at the desired temperature was subjected to a constant stress with a small oscillatory stress superposed. We compare our experimental results to a model designed to describe the linear and nonlinear mechanical properties of semi-flexible networks ${ }^{2,22}$. The model we use assumes no network relaxation over the timespan of the experiment. Chemically crosslinked networks commonly hold to this assumption, but for physically crosslinked gels this is not always the case. We experimentally verified by low-frequency rheological measurements that the PIC gel does not relax on a time scale 10 times the measurement time, even in very soft regimes, close to the gel point. This model describes $G_{0}$ and $\sigma_{c}$ quantitatively as a function of the polymer (or filament) concentration $c$ and persistence length $l_{\mathrm{p}}$, the temperature $T$ and the distance between crosslinks in the network $l_{c}$ :

$$
G_{0}=6 \rho k_{\mathrm{B}} T \frac{l_{\mathrm{p}}^{2}}{l_{\mathrm{c}}^{3}} \text { and } \sigma_{\mathrm{c}}=\rho k_{\mathrm{B}} T \frac{l_{\mathrm{p}}}{l_{\mathrm{c}}^{2}}
$$

where the polymer density in length per volume $\rho=\chi c / N$ and $\chi$ includes molecular constants ${ }^{16}, N$ is the number of polymer chains per bundle and $k_{\mathrm{B}}$ is Boltzmann's constant. PIC networks show a finite bundling behaviour in the gel (independent of concentration) and a thermal stiffening of the polymer chains. At our default gel concentrations $\left(c=0.1-5 \mathrm{mg} \mathrm{ml}^{-1}\right)$ where $l_{c}$ approximates the mesh size, equation (1) can be approximated as:

$$
G_{0}(T) \propto \sqrt{N^{3} c^{5}} k_{\mathrm{B}} T l_{\mathrm{p}, 0}^{2}(T) \text { and } \sigma_{\mathrm{c}}(T)^{2} k_{\mathrm{B}} T l_{\mathrm{p}, 0}(T)
$$

where $l_{\mathrm{p}, 0}$ is the persistence length of a single polymer chain rather than the bundle ${ }^{16}$.

Concentration dependence. The easiest method to change the mechanical properties of any hydrogel is to vary the concentration $c$ of the constituent polymer. A concentration study of $\mathbf{1 f}$ ( $L=158 \mathrm{~nm}$ ) reveals that, indeed, the plateau modulus $G_{0}$ and critical stress $\sigma_{\mathrm{c}}$ are strongly dependent on $c$ (Fig. 3). Plotting the differential modulus $K^{\prime}$ against stress as a function of concentration (Fig. 3a) clearly shows for all samples a linear regime at low $\sigma$ and a nonlinear regime at higher $\sigma$. In addition, the absolute values of $G_{0}$ and $\sigma_{\mathrm{c}}$ increase with $c$; scaling analysis shows a square dependence with $c$ for both (Fig. 3b), which corresponds to theoretical and literature values ${ }^{2,22,23}$. The curves collapse to a master curve when scaled against $G_{0}$ and $\sigma_{\mathrm{c}}$ (Fig. 3c). The plot suggests that a stiffening index $m=3 / 2$ is reached only at high stresses, as was observed previously at elevated temperatures ${ }^{16}$. The $3 / 2$ exponent is the upper limit for $m$ and is associated to enthalpically stretching a polymer chain along its length. Data recorded at $25^{\circ} \mathrm{C}$, only a few degrees away from $T_{\text {gel }}$, however, showed lower stiffening indices. For all concentrations, $m \approx 1$ close to $\sigma_{\mathrm{c}}$ (blue line Fig. $3 \mathrm{c}$ and blue squares Fig. 3d), while $m$ increases at higher stresses. Hence, at a specific stress, for instance $\sigma=10 \mathrm{~Pa}$ (red line Fig. 3a), $m$ decreases for samples with increasing $\sigma_{\mathrm{c}}$ (closer to $10 \mathrm{~Pa}$ ) and thus with increasing concentration (red circles Fig. 3d). In other words, not only does the gel become less mechanically sensitive at higher concentration, also its stress response becomes weaker.

This seemingly straightforward series of experiments yields two important insights. First, at high stress all gels have similar stiffnesses, nearly independent of the concentration. This is particularly important for biomedical applications of hydrogels, where both morphology and stiffness are key for their application. When cells stiffen their own matrix ${ }^{13}$, an increase in concentration may not contribute to an increase in the mechanical (micro)environment. Second, the concentration as a variable equally affects the plateau modulus and the critical stress, 

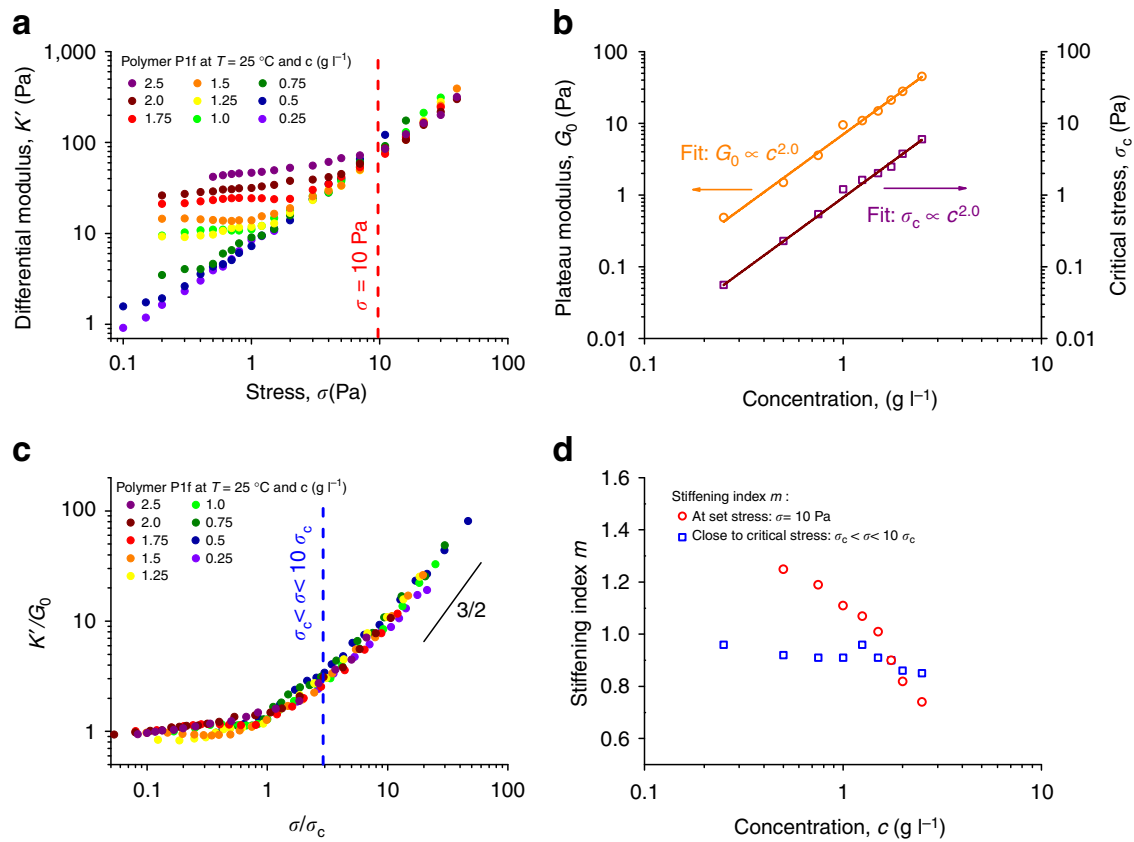

Figure 3 | Mechanical properties of PIC hydrogels $\left(\mathbf{P 1 f}, \mathbf{T}=\mathbf{2 5}^{\circ} \mathbf{C}\right.$ ) as a function of polymer concentration. (a) Differential modulus against stress at different polymer concentrations. Note that the $y$-axis spans 3 orders of magnitude in stiffness with a concentration range of only 1 order of magnitude. The dashed red line at $\sigma=10 \mathrm{~Pa}$ indicates where $m$ was determined. (b) Plateau modulus $\mathrm{G}_{0}$ (orange) and critical stress $\sigma_{\mathrm{c}}$ (dark red) against concentration (c). The solid lines are power law fits and show that both scale with $c^{2.0}$. (c) Scaling $K^{\prime}$ with $G_{0}$ and $\sigma$ with $\sigma_{\mathrm{c}}$ causes a collapse of the data to a single master curve that shows $K^{\prime} \propto \sigma^{3 / 2}$ only at high $\sigma$. The blue dashed line shows the point close to $\sigma_{\mathrm{c}}$ where $m$ was determined. (d) Stiffening index $m$ as a function of concentration. When determined close to $\sigma_{\mathrm{c}}$ (blue squares), $m$ is similar for all concentrations; when determined at a given stress (red circles, $\sigma=10 \mathrm{~Pa}$ ), $m$ is higher for lower concentration gels (since at lower $c, \sigma_{\mathrm{c}}$ is much lower and thus $\sigma-\sigma_{\mathrm{c}}$ will be higher). Note that the concentration axes $(\mathbf{b}, \mathbf{d})$ have logarithmic scales.

that is, hydrogels with higher elastic moduli inevitably become less sensitive to stress. This renders concentration a relatively ineffective tool to flexibly design the mechanical properties of a gel.

Chain length dependence. Another readily accessible variable available to polymer chemists is the molecular weight or the length of the polymers. Polymers 1a-1g, obtained by polymerizations with different monomer/catalyst ratios have contour lengths $L=20-180 \mathrm{~nm}$ (Table 1) with similar chain length distributions (Supplementary Fig. 1). We experimentally investigated the effect of the polymer chain length on the linear and nonlinear mechanical properties of the hydrogels, keeping the concentration of the polymers constant at $c=1 \mathrm{mg} \mathrm{ml}^{-1}$ (Fig. 4).

For covalently crosslinked gels of either flexible or semi-flexible polymers, the critical length scale that determines the stiffness is not the chain length, but the length between crosslinks $l_{c}$, which is predominantly controlled by the polymer concentration and the crosslink density. Recent computational simulations on semiflexible polymer gels, however, indicated that the gel stiffness will also depend on chain contour length $L$ for relatively short and flexible polymers, while for longer or stiffer polymers no dependency was observed ${ }^{24}$. Experimentally, we observe that the PIC gels require a minimum length to form gels of sufficient strength to investigate linearly or nonlinearly. Gels of polymer P1a were too weak to measure reliably, and gels of $\mathbf{P 1 b}$ ruptured at increased stress. Polymers P1c-P1g, however, show a clear linear and nonlinear regime (Fig. $4 \mathrm{a}$ ), where both the (linear) stiffness $G_{0}$ and critical stress $\sigma_{\mathrm{c}}$ increase with increasing $L$. Again, at high stress, all polymer gels behave similarly. With the polymer length, also the gelation temperature changes (Fig. 4b), where the shorter polymers have an increasing gel temperature. The increase in $T_{\text {gel }}$, is tentatively attributed to a delayed bundling process. At three different temperatures (dashed lines in Fig. 4b), $G_{0}$ (Fig. 4c) and $\sigma_{\mathrm{c}}$ (Fig. $4 \mathrm{~d}$ ) were recorded for the different length polymers. The observed dependencies: $G_{0} \propto L^{2}$ and $\sigma_{\mathrm{c}} \propto L$ are in perfect agreement with theoretical predictions for relatively short semi-flexible polymers ${ }^{24}$. Only good fits, however, are obtained when a minimum cut-off length $L_{\min } \approx 35 \mathrm{~nm}$ is considered, indicating a lower length limit for efficient bundle formation. We emphasize that $L_{\min }$ is unrelated to the mesh size of the gel at this concentration, which for these materials is 3-4 times larger. The inability of P1a (with average polymer length $L=34 \mathrm{~nm}$ ) to form hydrogels supports the model of a minimum required polymer length. The different scaling response to $G_{0}$ and $\sigma_{\mathrm{c}}$ makes $L$ (compared to $c$ ) a far more effective parameter to tune the mechanical properties of a gel.

Temperature dependence. The largest change in properties in PIC-based gels is thermally induced. The PIC polymers form lowviscosity solutions at low temperatures and elastic gels when heated beyond their gelation temperature. Hydrophobic interactions of the oligoglycol substituents are responsible for this lower critical solution temperature behaviour, which has also been observed in other polymers ${ }^{20}$. All mechanical properties change drastically in the (reversible) transition from a liquid to a hydrogel and we will discuss three regimes separately: in the gel (high T), in the transition and just below the transition (low T). The overall mechanical properties show that on increasing the temperature both the stiffness and the critical stress increase (Fig. 5a). At high stress, however, the data at different temperatures all collapse to a single curve, similar as was the case in the concentration and length studies. 

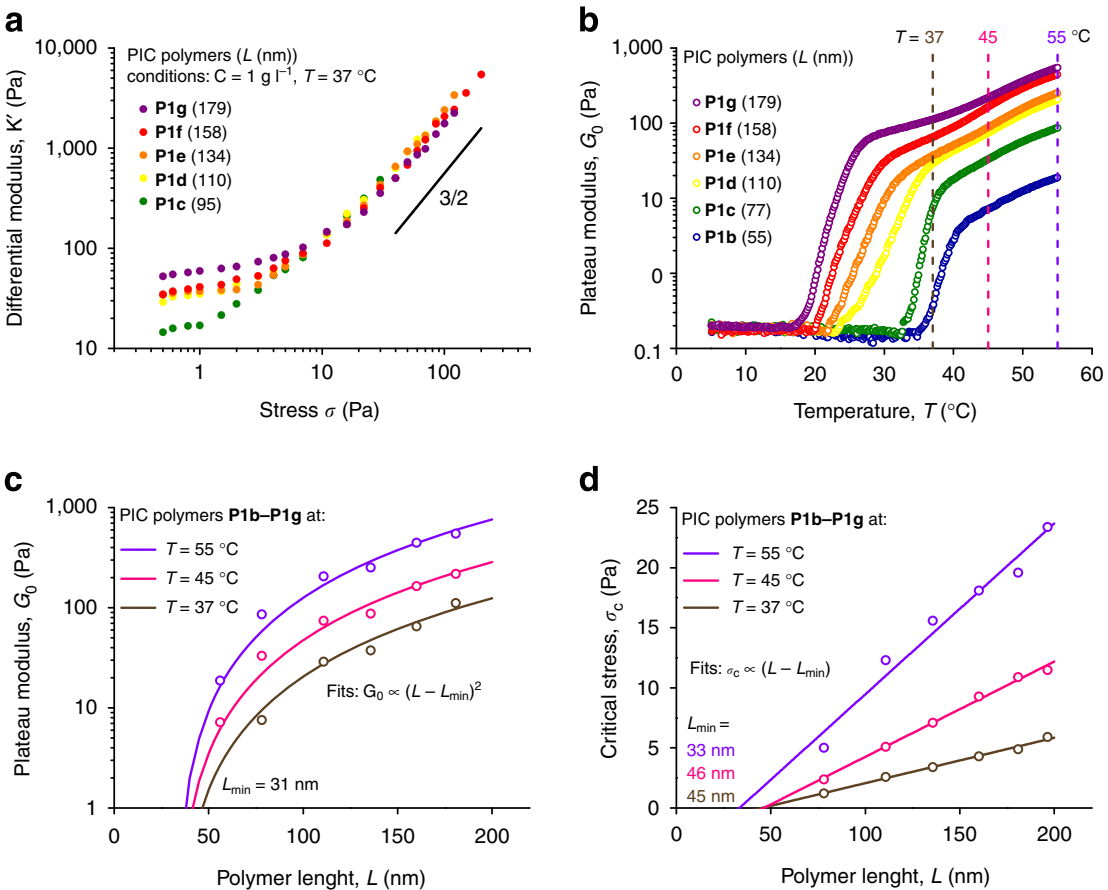

Figure 4 | Molecular weight dependence of the mechanical properties of the P1b-P1g hydrogels (at $\mathbf{c}=\mathbf{1} \mathbf{m g ~ m l}^{-1}$ ). (a) Differential modulus against stress for different molecular weight or length $L$ polymers at $T=37^{\circ} \mathrm{C}$. The solid line shows the high stress limit $m=3 / 2$. (b) Plateau modulus $G_{0}$ as a function of temperature for gels of different length polymers. (c) Data extracted from $\mathbf{b}$ : $G_{0}$ as a function of $L$ at $T=37,45$ and $55^{\circ} \mathrm{C}$. The solid line shows quadratic fits to $\left(L-L_{\mathrm{min}}\right)$, where fitting parameter $L_{\min }$ represents a minimum polymer length (see main text). (d) The critical stress $\sigma_{\mathrm{c}}$ as a function of $L$ at the same temperature. Here the solid lines are linear fits to $\left(L-L_{\min }\right)$.

The stiffness $G_{0}$ in the elastic gel regime shows a dual thermal response (Fig. 5b). Network theory predicts a linear dependence on $T$, associated with thermal fluctuations of the polymer chains. When the thermal fluctuations increase, it becomes more difficult to stretch the polymer chains and hence, the stiffness increases ${ }^{22}$. A second, exponential contribution originates from the stiffening of the polymer chains (increase in $l_{\mathrm{p}, 0}$ ) themselves with $T$, as was established earlier for PIC gels ${ }^{16}$. Both factors together contribute with:

$$
G_{0}(T) \propto k_{\mathrm{B}} T l_{\mathrm{p}, 0}^{2}(T) \propto T e^{2 \beta T}
$$

For P1f, we find $\beta \approx 0.084 \mathrm{~K}^{-1}$, which means that the gel stiffness doubles every $\sim 4{ }^{\circ} \mathrm{C}$. In and below the gelation transition, $G_{0}$ decreases rapidly until at low temperatures it becomes difficult to measure $G_{0}$ accurately due to the limitations of our experimental set-up.

The critical stress similarly depends on $T$ and $l_{\mathrm{p}, 0}(T)$ and is expected to scale as $k_{\mathrm{B}} T e^{\beta T}$, as was also observed experimentally (Fig. $5 c$, pink data) with a similar exponent $\beta=0.088 \mathrm{~K}^{-1}$. The temperature increases the stiffness more than the critical stress, which results in a decrease in the critical strain $\gamma_{c}$ with $T$ :

$$
\gamma_{\mathrm{c}}(T)=\frac{\sigma_{\mathrm{c}}(T)}{G_{0}(T)} \propto e^{-\beta T}
$$

Indeed, we see experimentally (Fig. 5c, blue data) that at elevated temperatures only very small deformations $\left(\gamma_{c, T=37{ }^{\circ} \mathrm{C}}=8 \%\right)$ are required to enter the nonlinear, responsive regime, while at lower $T$ these deformations must be much higher $\left(\gamma_{c, T=22{ }^{\circ} \mathrm{C}}=25 \%\right)$. The absolute value of the critical strain in a semi-flexible gel can further be tuned by the polymer length $L$, where for increased $L$ a decrease in $\gamma_{c}$ is expected based on equation (4). Again, changing the polymer concentration will be less effective as both $\sigma_{\mathrm{c}}$ and $G_{0}$ scale similarly with $c$ (Fig. 3b).
The exponential decrease in $\gamma_{\mathrm{c}}$ given by $\beta$, however, is a molecular property that cannot be altered by changing $L$ or $c$. In contrast to $G_{0}, \sigma_{c}$ and $\gamma_{c}, m$ is only weakly temperature dependent in the gel regime (Fig. 5d).

We also measured the mechanical properties of the aqueous materials during the gelation transition $\left(T=19-22^{\circ} \mathrm{C}\right)$ and even before gelation, when the material is a low-viscous dilute polymer solution $\left(T=17-19^{\circ} \mathrm{C}\right)$. At even lower temperatures, instrument inertia dominates our rheological data, which renders our results unreliable. In the entropically driven phase transition, the connectivity (that is, the number of crosslinks) of the network will rapidly change as a function of $T$. This causes $G_{0}$ to increase by a factor of 10 over only $5^{\circ} \mathrm{C}$ (Fig. $4 \mathrm{~b}$ ) and $\sigma_{\mathrm{c}}$ even more (Fig. 4c). As a result, $\gamma_{c}$ shows a maximum precisely at the temperature where the network is fully established. This is an important factor in designing soft, highly strainable networks, as the stiffness of the materials rapidly increases when strained beyond the critical strain.

The stiffening index $m$, the strength of the mechanical response to stress shows two additional regimes. When cooling from $22^{\circ} \mathrm{C}$, it rapidly decreases to $m \approx 0.6$. By decreasing the connectivity in a network, simulations have found anomalous behaviour at the critical point, which is the connectivity at which the network becomes mechanically floppy. This point, called the marginal point, is predicted to show a decreased stiffening index $(m=0.5)$. So far, marginal materials have been described only in theory and by simulations ${ }^{17}$ and no experimental (bio)polymer network has validated these predictions yet. The PIC gels are the first materials that display some of the predicted characteristics ${ }^{25}$. An additional prediction is a vanishing $\sigma_{\mathrm{c}}$ (also experimentally observed in the PIC hydrogel, Fig. 5c), which makes the material in and around the marginal point extremely stress sensitive and allows the design of materials that in a small stress (or strain) regime can span 2 or 3 orders of magnitude in stiffness. 
a



C

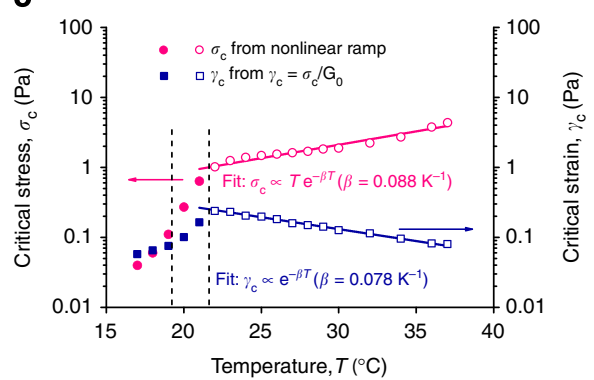

b

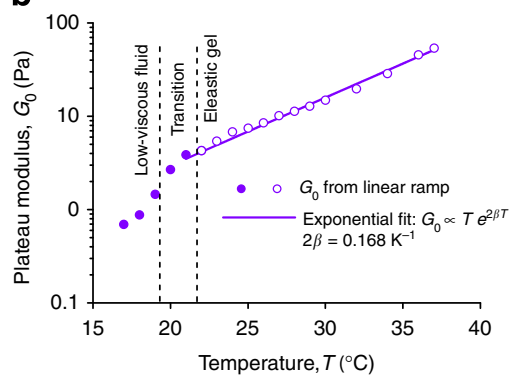

d

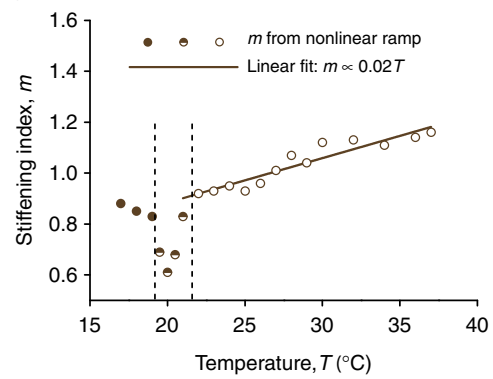

Figure 5 | Temperature dependence of the mechanical properties of the P1f hydrogel (at $\mathbf{1 ~} \mathbf{~ g ~}_{\mathbf{~ m l}} \mathbf{l}^{\mathbf{1}}$ ). (a) Differential modulus against stress at different temperatures. The solid line shows the high stress limit $m=3 / 2$. (b) Plateau modulus $G_{0}$, as a function of temperature $T$ (linear scale). In the gel at $T \geq 22^{\circ} \mathrm{C}, G_{0}$ increases exponentially with $T$ over the investigated temperature window; the solid line is a fit to $T e^{2 \beta T}$ with $\beta \approx 0.08 \mathrm{~K}-1$. At $T \leq 22{ }^{\circ} \mathrm{C}, \mathrm{G}_{0}$ increases fast with $T$, corresponding to the transition from liquid to gel. (c) critical stress $\sigma_{\mathrm{c}}$ (pink circles) and critical strain $\gamma_{\mathrm{c}}$ (blue squares) as a function of $T$; the solid lines are fits to $T e^{\beta T}$ and $e^{-\beta T}$ with $\beta \approx 0.08 \mathrm{~K}^{-1}$. Both $\sigma_{\mathrm{c}}$ and $\gamma_{\mathrm{c}}$ show a sharp decrease at $T \leq 22^{\circ} \mathrm{C}$. (d) The stiffening index $m$ as a function of $T$ clearly shows three regimes. In the high temperature regime, $m$ weakly scales linearly with $T$, and around the marginal regime (see text) $m$ sharply decreases while it restores to 0.9 on further decreasing $T$. The values for $m$ were recorded at stresses close to the critical stress (at $\sigma=3 \sigma_{c}$ ). At higher stress, in particular at high temperature, $m$ will approach 1.5 ref. 16, but such stresses are impossible to apply close to $T_{\text {gel. }}$.

Upon further lowering $T, m$ increases again to $\sim 0.9$. In this 'pre-marginal' regime, no continuous network is yet formed, but stress applied to the semi-flexible polymer chains induces network formation. Simulations show that $m$ should reach unity eventually, but we were unable to experimentally probe deeper in the pre-marginal regime. The material at this temperature range has extremely interesting properties. At low stress or deformation, it behaves as a fluid; it can be poured from a vial. Rheologically, we see that the viscous properties dominate the elastic properties (Supplementary Fig. 2). However, as the nonlinear strainstiffening behaviour is still present in the material, the elasticity increases steeply with stress. Since, in addition, the critical stress is very small $\left(\sigma_{c, T=17^{\circ} \mathrm{C}}<0.05 \mathrm{~Pa}\right)$, the threshold for stiffening is virtually absent and the solution transforms into a fully elastic solid at very low stress. Finally, the relatively high stiffening index in this regime causes the stiffness to increase rapidly on further increasing the stress. Even at relatively low stresses, for example, at $1 \mathrm{~Pa}, K^{\prime}$ already increased by an order of magnitude. This makes marginal, but even more so pre-marginal, 'gels' or solutions extremely sensitive and also very responsive to stress. Such materials could also be interesting for tissue engineering applications when one considers that cells can easily apply forces of such magnitude to their environment, and thus can manipulate the local mechanical properties of their matrix.

\section{Discussion}

The controlled manipulation of the linear and nonlinear mechanical properties of a hydrogel is a complex task. Changing a variable such as concentration affects all three mechanical parameters $G_{0}, \sigma_{\mathrm{c}}$ and $m$. In addition, important biological parameters, such as pore size also change. This makes it difficult to make appropriate comparisons within a series, let alone between series. A thorough study of these different dependencies, however, allows one to profit from this complex behaviour. As an example, the stronger increase of $G_{0}$ (quadratic) compared with $\sigma_{\mathrm{c}}$ (linear) with $L$ presents an opportunity to develop specific materials properties, such as series of materials with constant stiffness, but with different sensitivity (and response) to deformation. When keeping $L \times c$ constant, $G_{0}$ remains constant, but $\sigma_{\mathrm{c}}$ varies with their ratio (Fig. 6). Changing the value of $L \times c$ results in different (constant) linear moduli, but in the same overall behaviour. Analogously, one can prepare series of materials with the same critical stress (keeping $\sqrt{L} \times c$ constant) but by varying linear modulus. This example of a combined length-concentration approach allows for full control on stiffness and mechanical sensitivity in soft matter, much more than what would be possible with a single variable. One can easily imagine, however, that also other combinations of variables can be used to obtain mechanical control at a similar level.

Interestingly, for sufficiently stressed gels, we see that for the concentration, length and temperature series, these dependencies disappear and nearly universal behaviour emerges. Considering that cells can effectively apply stresses up to $10 \mathrm{~Pa}$ to their local environment ${ }^{3,13}$, it is even more intriguing to see that for PIC gels and for many biological gels, this 'universal' transition occurs well into the biologically accessible range. This may have implications for how we currently consider the role of mechanics in, for instance, tissue engineering. Cells may be able to control their own micromechanical environment by adhering to the matrix and actively stretching it. The observed universal behaviour of variables such as, in particular, concentration but also polymer length suggests that these parameters are ineffective for mechanical control, but merely decrease the porosity of the 


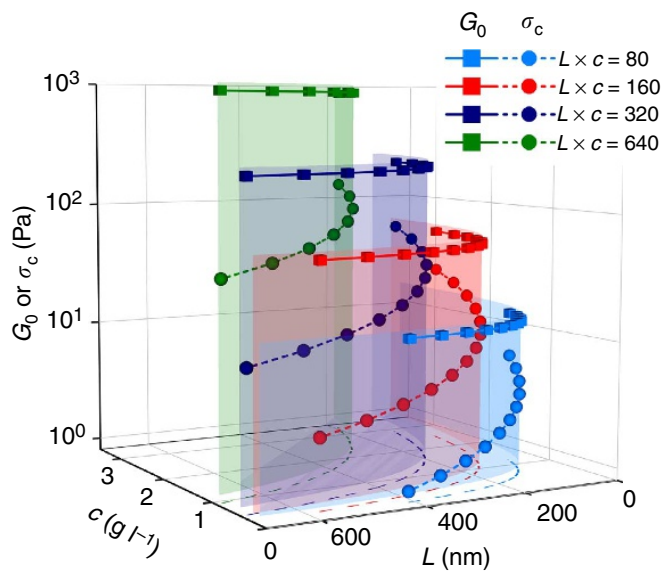

Figure 6 | Interpolated mechanical properties of gels with constant modulus and varying sensitivity to stress. When in a series of gels the product $L \times c$ and $G_{0}$ remain constant, but $\sigma_{\mathrm{c}}$ changes. Here we selected four series of constant $G_{0}$ in the range $10-1,000 \mathrm{~Pa}$ (squares) and $\sigma_{\mathrm{c}}$ varying in each series (circles). The projection of the curves on the $x-y$ plane (dashed lines) shows the corresponding length and concentrations.

matrix, potentially reducing efficient nutrient transport and inhibiting cell spreading and proliferation. This supports the idea that, in fact, cells may probe different material properties than those which one characterizes with straightforward linear mechanical analysis ${ }^{11,12}$.

In conclusion, we described the effect of common, easily controllable variables (concentration, polymer length and temperature) on the mechanical properties of semi-flexible polymer hydrogels. Such gels are highly strain stiffening and we can use this property to create extremely stress-sensitive materials. We anticipate that the behaviour that we described is ubiquitous, in other words, many other strain-stiffening materials will behave similarly. Why, then, were these properties never described before? Two major reasons: (1) the majority of strain-stiffening materials have biological origins and it is often not easy to precisely define and control critical parameters such as length or connectivity, and (2) the length scales in the materials should match to enter the highly responsive regime: both the length $(L)$ and the stiffness of the polymer bundle or filament $\left(l_{\mathrm{p}}\right)$ should be of the same order of magnitude as the mesh size of the network. When the polymers are too flexible ( $l_{\mathrm{p}}$ is too low), linear behaviour dominates and stiffening will only be observed at high stresses where the gel is likely to break. In addition, a (significant) minimum length is required to maintain network properties (sufficient crosslinks) at low concentrations. Typically, the persistence length of synthetic polymers is too low, even when we consider them to be rigid polymers. We firmly believe that bundle formation is the best way forward to increase $l_{\mathrm{p}}$, but full control over such process remains a challenge to be solved. The outlook, however, is a class of materials where the mechanical properties $\left(G_{0}, \sigma_{\mathrm{c}}\right.$ and $\left.m\right)$ can be readily adjusted by changing the conditions ( $c, L, T$, and more), but also can be dramatically and immediately altered as a function of stress and/or other external stimuli. Such tremendous level of control will be highly beneficial for the biomedical field as well as other fields that use responsive soft matter applications.

\section{Methods}

Polymer synthesis and characterization. The isocyanide monomer ${ }^{18}$ was dissolved in freshly distilled toluene $\left(50 \mathrm{mg} \mathrm{ml}^{-1}\right)$ and stirred for several minutes. A solution of $\mathrm{Ni}\left(\mathrm{ClO}_{4}\right)_{2} \cdot 6 \mathrm{H}_{2} \mathrm{O}\left(0.1 \mathrm{mg} \mathrm{ml}^{-1}\right)$ in freshly distilled toluene/ethanol (9:1) was added at once. The mixture was stirred for $24 \mathrm{~h}$ at room temperature under air. The resulting polymer was precipitated in diisopropyl ether with vigorous stirring and collected by filtration. The solid was redissolved in dichloromethane and again precipitated in diisopropyl ether and collected by filtration. This procedure was repeated one more time and the polymer was dried under vacuum to yield the polymer as a yellow solid in $60-70 \%$ yield. The molecular weight of the polymer was characterized by viscometry and AFM, as shown in Supplementary Information. For viscometry measurements, the polymer was dissolved in acetonitrile $\left(0.02-6.0 \mathrm{mg} \mathrm{ml}^{-1}\right)$ and the intrinsic viscosity of these solutions was measured at a temperature of $25^{\circ} \mathrm{C}$. The average viscosity molecular weight, $M_{\mathrm{v}}$, of the polymers was calculated using the empirical Mark-Houwink equation, $[\eta]=K M_{\mathrm{v}}^{a}$, where $[\eta]$ is the intrinsic viscosity of the polymer solution as determined from viscometry measurements (Supplementary Fig. 3), and MarkHouwink parameters $K$ and $a$ depend on polymer and solvent characteristics. We used values that were previously determined for (other) rigid polyisocyanides ${ }^{26}$ : $K=1.4 \times 10^{-9}$ and $a=1.75$. For AFM measurements, the polymer was dissolved in freshly distilled dichloromethane $\left(10^{-3} \mathrm{mg} \mathrm{ml}^{-1}\right)$. This polymer solution was spin coated onto a freshly cleaved Muscovite Mica surface at 3,000 r.p.m. AFM measurements were performed using a dimension 3100 microscope operated with a nanoscope IV control unit (Digital Instruments). All images were recorded with the AFM operating in Tapping Mode in air at room temperature, with a resolution of $1,024 \times 1,024$ pixels. Commercial tapping-mode golden-coated silicon tips (NTMDT) were used with a typical resonance frequency around $300 \mathrm{kHz}$. Polymer lengths were evaluated using ImageJ software.

Rheology. Rheological measurements were carried out with a stress-controlled rheometer (Discovery HR-1, TA Instruments) in an aluminium parallel plate geometry ( $40 \mathrm{~mm}$ diameter) with a gap of $500 \mu \mathrm{m}$ in a temperature-controlled environment. To probe the linear regime $\left(G_{0}\right)$, the sample was heated to the desired temperature, and after a short waiting period for equilibration the complex modulus $G^{*}$ was determined by applying an oscillating deformation of amplitude $\gamma=0.01$ in a frequency sweep of $\omega=10-0.1 \mathrm{~Hz}$. The nonlinear regime $\left(\sigma_{\mathrm{c}}\right.$ and $\left.m\right)$ was studied using a pre-stress protocol, where the sample, at the desired temperature was subjected to a constant stress $\sigma_{0}$ with a small oscillatory stress superposed, also at $\omega=10-0.1 \mathrm{~Hz}$. In the pre-stress protocol that we use to probe the nonlinear mechanical regime, we apply a constant stress to the material. We did not find relaxation processes in the materials, even at high stresses.

\section{References}

1. Storm, C., Pastore, J. J., MacKintosh, F. C., Lubensky, T. C. \& Janmey, P. A. Nonlinear elasticity in biological gels. Nature 435, 191-194 (2005).

2. Gardel, M. L. et al. Elastic behavior of cross-linked and bundled actin networks Science 304, 1301-1305 (2004).

3. Rudnicki, M. S. et al. Nonlinear strain stiffening is not sufficient to explain how far cells can feel on fibrous protein gels. Biophys. J. 105, 11-20 (2013).

4. Thiele, J., Ma, Y., Bruekers, S. M. C., Ma, S. \& Huck, W. T. S. 25th Anniversary article: designer hydrogels for cell cultures: a materials selection guide. $A d v$. Mater. 26, 125-148 (2014).

5. Place, E. S., Evans, N. D. \& Stevens, M. M. Complexity in biomaterials for tissue engineering. Nat. Mater. 8, 457-470 (2009).

6. Discher, D. E., Janmey, P. \& Wang, Y. L. Tissue cells feel and respond to the stiffness of their substrate. Science 310, 1139-1143 (2005).

7. Engler, A. J., Sen, S., Sweeney, H. L. \& Discher, D. E. Matrix elasticity directs stem cell lineage specification. Cell 126, 677-689 (2006).

8. Guvendiren, M. \& Burdick, J. A. Stiffening hydrogels to probe short-and longterm cellular responses to dynamic mechanics. Nat. Commun. 3, 792 (2012).

9. Wen, J. H. et al. Interplay of matrix stiffness and protein tethering in stem cell differentiation. Nat. Mater. 13, 979-987 (2014).

10. Chaudhuri, O. et al. Extracellular matrix stiffness and composition jointly regulate the induction of malignant phenotypes in mammary epithelium. Nat. Mater. 13, 970-978 (2014).

11. Wen, Q. \& Janmey, P. A. Effects of non-linearity on cell-ECM interactions. Exp. Cell Res. 319, 2481-2489 (2013).

12. Winer, J. P., Oake, S. \& Janmey, P. A. Non-linear elasticity of extracellular matrices enables contractile cells to communicate local position and orientation. PLoS ONE 4, e6382 (2009).

13. Jansen, K. A., Bacabac, R. G., Piechocka, I. K. \& Koenderink, G. H. Cells actively stiffen fibrin networks by generating contractile stress. Biophys. J. 105, 2240-2251 (2013).

14. Motte, S. \& Kaufman, L. J. Strain stiffening in collagen I networks. Biopolymers 99, 35-46 (2013)

15. Broedersz, C. P. \& MacKintosh, F. C. Modeling semiflexible polymer networks. Rev. Mod. Phys. 86, 995-1036 (2014).

16. Kouwer, P. H. J. et al. Responsive biomimetic networks from polyisocyanopeptide hydrogels. Nature 493, 651-655 (2013).

17. Broedersz, C. P., Mao, X. M., Lubensky, T. C. \& MacKintosh, F. C. Criticality and isostaticity in fibre networks. Nat. Phys. 7, 983-988 (2011).

18. Koepf, M. et al. Preparation and characterization of non-linear poly(ethylene glycol) analogs from oligo(ethylene glycol) functionalized polyisocyanopeptides. Eur. Polym. J. 49, 1510-1522 (2013). 
19. Cornelissen, J. J. L. M. et al. $\beta$-Helical polymers from isocyanopeptides. Science 293, 676-680 (2001).

20. Weber, C., Hoogenboom, R. \& Schubert, U. S. Temperature responsive biocompatible polymers based on poly(ethylene oxide) and poly(2-oxazoline)s. Prog. Polym. Sci. 37, 686-714 (2012).

21. Piechocka, I. K., Bacabac, R. G., Potters, M., MacKintosh, F. C. \& Koenderink, G. H. Structural hierarchy governs fibrin gel mechanics. Biophys. J. 98, 2281-2289 (2010).

22. MacKintosh, F. C., Kas, J. \& Janmey, P. A. Elasticity of semiflexible biopolymer networks. Phys. Rev. Lett. 75, 4425-4428 (1995).

23. Lin, Y. C. et al. Origins of elasticity in intermediate filament networks. Phys. Rev. Lett. 104, 058101 (2010).

24. Broedersz, C. P., Sheinman, M. \& Mackintosh, F. C. Filament-length-controlled elasticity in 3D fiber networks. Phys. Rev. Lett. 108, 078102 (2012).

25. Dennison, M. et al. Critical behaviour in the elastic response of hydrogels. Preprint at http://arxiv.org/abs/1407.0543 (2014).

26. Van Beijnen, A., Nolte, R., Drenth, W., Hezemans, A. \& Van de Coolwijk, P. Helical configuration of poly(iminomethylenes). Screw sense of polymers derived from optically active alkyl isocyanides. Macromolecules 13, 1386-1391 (1980).

\section{Acknowledgements}

We acknowledge financial support from NRSCC (M.J., A.E.R), FOM/NWO (M.D., F.C.M), NWO Gravitation (grant 024.001.035) and NanoNextNL (A.E.R., P.H.J.K.)

\section{Author contributions}

M.J. and M.F.J.M. synthesized the materials; M.D. performed simulation studies. M.J. and P.H.J.K. designed, conducted and interpreted the rheological experiments. F.C.M., A.E.R. and P.H.J.K. supervised the project. All authors contributed to the manuscript.

\section{Additional information}

Supplementary Information accompanies this paper at http://www.nature.com/ naturecommunications

Competing financial interests: The authors declare no competing financial interests.

Reprints and permission information is available online at http://npg.nature.com/ reprintsandpermissions/

How to cite this article: Jaspers, M. et al. Ultra-responsive soft matter from strain-stiffening hydrogels. Nat. Commun. 5:5808 doi: 10.1038/ncomms6808 (2014).

\section{(c) (i)}

This work is licensed under a Creative Commons Attribution 4.0 International License. The images or other third party material in this article are included in the article's Creative Commons license, unless indicated otherwise in the credit line; if the material is not included under the Creative Commons license, users will need to obtain permission from the license holder to reproduce the material. To view a copy of this license, visit http://creativecommons.org/licenses/by/4.0/ 